\title{
Assessment of Genetic Diversity of Freshwater Mud Eel (Monopterus cuchia) Using RAPD and RFLP Markers
}

\author{
Md. Faruque Miah ${ }^{1 *}$, Saeed Anwar ${ }^{1,2}$, Md. Hazrat Ali ${ }^{1}$, Niamul Naser $\mathbf{M}^{3}$ and Kawser Ahmed
}

${ }^{1}$ Department of Genetic Engineering and Biotechnology, School of Life Sciences, Shahjalal University of Science and Technology, Sylhet, Bangladesh ${ }^{2}$ Research and Development Department, Bangladesh Science Society, Nikunja, Dhaka, Bangladesh

${ }^{3}$ Faculty of Biological Sciences, Department of Zoology, University of Dhaka, Dhaka, Bangladesh

${ }^{4}$ Faculty of Earth and Environmental Sciences, Department of Oceanography, University of Dhaka, Dhaka, Bangladesh

\begin{abstract}
Background: Monopterus cuchia, an economically important eel of Bangladesh, is confronting the challenge of population reduction and germplasm degeneration since the vast majority of fingerlings are collected from natural habitats and artificial cultivation of the fish is yet to be established.

Materials and Methods: 30 individuals of $M$. cuchia from a natural population of Northern-East part of Bangladesh were analyzed using 8 decamer primers and glutamine synthetase gene was digested with 2 restriction enzymes.

Results: A total of 735 bands with 228 polymorphic loci were detected among the selected 30 individuals by using RAPD assay while $100 \%$ polymorphism was revealed by all the primers. The genetic distance among the individuals was calculated by using the data from pair-wise similarity index where 36 groups of genetic diversities were measured while the lowest and highest genetic distance were found 0.58 and 0.97 respectively with an average genetic diversity of 0.81 . The Nei's genetic similarity values were found from 0.06 to 0.6 where average value was recorded 0.301746 which was found significant regarding genetic distance. Phylogenetic relationships using UPGMA clustering revealed linkage distance ranged from 3.6 to 6.24 and generated 6 clusters by 11 clades with the involvement of 22 individuals while the rest of the samples were connected to those clades with specific linkage distances. Genetic diversity of glutamine synthetase gene was analyzed with two restriction enzymes, e.g. + Cfrl and $+\mathrm{Hpy} 178 \mathrm{III}$ while both the enzymes digested the gene fragment at a length of $541 \mathrm{bp}$ and polymorphism was detected in terms of wild type homogygotes, polymorphic homozygote and heterozygosity. The genetic diversity was observed by using the RFLP band analysis and four different groups of individuals were identified with the p-values of $0,0.033,0.05$ and 1 respectively whereas different distances were found among the groups indicating the polymorphism among experimental individuals of glutamine synthetase gene.
\end{abstract}

Conclusion: Herein, RAPD and RFLP analysis indicating the rich genetic diversity of this fish in the experimental ecological habitat and this would come helpful for the conservation of germplasm diversity and to support the sustainable breeding program of M. cuchia.

Keywords: RAPD; RFLP; Glutamine synthetase; Monopterus cuchia; Genetic diversity

\section{Introduction}

Monopterus cuchia [1], also known as Gangetic mud eel, freshwater mud eel, Kuicha, or Kunche, [2] of synbranchidae family under the order of synbranchiformes $[3,4]$ is one of the common freshwater eels found in Bangladesh. Although the fish has high nutritional as well as medicinal values, it is consumed only by peoples of some tribes and few other castes [5]. The trade of the fish offers a great export fishery to at least 15 foreign countries, including China, Malaysia, Singapore, Japan and Taiwan and almost all the harvested fish are exported [6].The fish has the ability to survive in harsh conditions as it possess systems for both aquatic and aerial respiration [7] and also the ability to survive 90 to 132 days without having foods. These offers the culture of a large number of fishes in small tanks, aquarium and other vessels as well as the transportation to distant places using simple earthenware or plastic jars $[1,8]$.

Development of social fishery can help the socioeconomic welfare of an area in a unique way [9]. But due to overfishing as well as water obstruction, destruction of habitat, water pollution, emergence of diseases etc. the populations of M. cuchia in Bangladesh is falling-off at an alarming rate $[10,11]$ and consequently, it is recorded as a least concerned species by IUCN [12]. Careful monitoring and research have become essential for its existence in the natural bodies. Being commercially important and presumed as a low-cost enterprise to the poor farmers, freshwater mud eel can be a good choice for artificial culture to meet the increasing demand of animal protein in Bangladesh as well as to earn foreign currencies [13] to help the development of our national economy.

As it has been mentioned earlier, $M$. cuchia can tolerate harsh conditions, its culture is easier and highly profitable than a number of other small size fish culture activities [14,15]. Several techniques of capturing the fish have been established, however, no cultural practice has been consecrated and only a few works have from different aspects been done on this so far $[6,5,16-22]$. The mud eel has also been identified for polyculture in seasonal and perennial ponds as well as in paddy fields, and in Bangladesh, some experiments were performed in captivity for observing growth, survival and diet condition [23].

*Corresponding author: Md. Faruque Miah, Department of Genetic Engineering and Biotechnology, School of Life Sciences, Shahjalal University of Science and Technology, Sylhet 3114, Bangladesh, Tel: +8801712865321; E-mail: faruque-btc@sust.edu

Received May 27, 2018; Accepted July 23, 2018; Published July 28, 2018

Citation: Md. Miah F, Anwar S, Md. Ali H, Naser MN, Ahmed K (2018) Assessment of Genetic Diversity of Freshwater Mud Eel (Monopterus cuchia) Using RAPD and RFLP Markers. J Aquac Res Development 9: 543. doi: 10.4172/2155-9546.1000543

Copyright: ( 2018 Miah F Md, et al. This is an open-access article distributed under the terms of the Creative Commons Attribution License, which permits unrestricted use, distribution, and reproduction in any medium, provided the original author and source are credited. 
In order to develop a scientifically proved sustainable eel culture system, it is necessary to produce huge amounts of fries artificially while unsuccessful induced breeding was observed without spawning responses [24,25]. The unprosperous reports regarding induced breeding of the fish refer to the necessity of the studies to be conducted on the genetic characterization of the fish to obtain reliable seeds for aquaculture and to develop a sustainable selective breeding program. But, unfortunately, so far, no large-scale works have been found on the genetic characterization of $M$. cuchia barring some tiny DNA fingerprinting analysis using RAPD markers in China [4] and in Bangladesh [26-28].

Understanding the genetic diversity at molecular level is a prerequisite in developing a sustainable eel culture system along with effective conservation and utilization of $M$. cuchia genetic resources, and, in this circumstance, this current study represents a novel genetic survey investigating the genetic status of $M$. cuchia in Bangladesh. Genetic diversity is considered as a key tool for the development of breeding programs and conservation of species and in this study, we have assessed the genetic diversity of $M$. cuchia using RAPD (Random Amplified Polymorphic DNA) technique. Additionally, we have observed the genetic diversity of Glutamine Synthatase (GS) gene using PCR-RFLP technique, which is involved in the detoxification of ammonia and enabling the fish to survive in the extreme conditions and regarded as an important player for analysis of breeding biology of the fish $[29,30]$. We studied the genetic diversity and relationship among the M. cuchia individuals collected from the Hakaluki Haor (an aquatic ecosystem), a natural habitat of the fish in Bangladesh. Taking into account that, the fish possess high export demand and excellent nutritional values, the long-term goal of this work is to help the conservation of the species, sustainable commercial culture of it and also to tip an alternative livelihood for people involved in the fisheries sector [30].

\section{Research Methodology}

\section{Collection of sample fishes and identification}

Fishes were collected randomly by the help of the officials of regional office of Department of Fisheries, Government of Peoples Republic of Bangladesh and professional fish catchers from Hakaluki Haor (Haor $=$ a natural water body; $23^{\circ} 35^{\prime}-24^{\circ} 44^{\prime} \mathrm{N}, 92^{\circ} 01^{\prime}-92^{\circ} 09^{\prime}$ E) of NorthernEast part of Bangladesh. A total of 30 individuals were selected for the study. Collected fishes were identified using morphometric characteristics as described by Shafi and Quddus, Rahman, Talwar and Jhingran [31-33]. Collected fishes were transferred to the Animal Keeping Laboratory of the department of Genetic Engineering and Biotechnology (GEB), Shahjalal University of Science and Technology (SUST), Sylhet 3114, Bangladesh and were kept in live fish preservation tanks until sacrificed humanely for tissues to be isolated. The study was undertaken after approval from the Animal Care and Ethics Committee of SUST.

\section{Tissue isolation and DNA extraction}

Each sacrificed fish was dissected and liver tissue was isolated from each individual. Isolated tissues were washed using distilled water and 70\% alcohol and preserved separately in Eppendorfs in $100 \%$ alcohol at $-20^{\circ} \mathrm{C}$ temperature freezer cabin. The desired genomic DNA was extracted by using a commercially available kit, (Bioserve, CAT.No. 2025) and DNA quality was checked on $0.8 \%$ agarose by gel electrophoresis and using gel documentation system with digital camera (Panasonic DMC-fs20) image was captured. A good quality DNA was observed from each of the individual where $1 \mathrm{~kb}$ Plus DNA ladder (GeneRuler ${ }^{\mathrm{TM}}$ ) was used to compare the quality of the DNA. Finally, the extracted DNA of each sample was stored at $-20^{\circ} \mathrm{C}$. In order to perform PCR based examinations, a concentration of DNA around $200 \mathrm{ng} / \mu \mathrm{l}$ was required and hence, specimen with variable concentration of the DNA was adjusted to $\sim 200 \mathrm{ng} / \mu \mathrm{l}$ before used in PCR.

\section{PCR-RAPD assay}

Eight decamer primers, e.g. B-03 (CATCCCCCTG), OPF 14 (TGCTGCAGGT), C 04 (CCGCATCTAC), OPB 05 (TGCGCCCTTC), OPB 08 (GTCCACACGG), OPB 12 (CCTTGACGCA) OPB 19 (ACCCCCGAAG) and UBC 122 (GTAGACGAGC) (Operon Technologies Inc. USA) were adopted and utilized for RAPD assaying of freshwater mud eel. The PCR amplification was carried out in a total volume of $15 \mu \mathrm{l}$ containing $8 \mu \mathrm{l}$ of master mix (Promega Hot Start), $1 \mu \mathrm{l}$ of $20 \mu \mathrm{M}$ working solution of primer, $2 \mu \mathrm{l}$ of template DNA and $4 \mu \mathrm{l}$ deionized distilled water. After an initial 1-minute denaturation at $94^{\circ} \mathrm{C}$, followed by 30 cycles of $94^{\circ} \mathrm{C}$ denaturation for 1 minute, annealing for a minute at $30^{\circ} \mathrm{C}$ for $\mathrm{UBC} 122,32^{\circ} \mathrm{C}$ for $\mathrm{B}-03,33^{\circ} \mathrm{C}$ for OPF $14, \mathrm{C}-04$ and OPB05, and $34^{\circ} \mathrm{C}$ for OPB 08 , OPB 12 and OPB 19 and $72^{\circ} \mathrm{C}$ extension for 2 minutes. A final 7 -minute extension at $72^{\circ} \mathrm{C}$ completed the reaction.

\section{RAPD data analysis}

Different formulae, calculations and software were used for analyzing RAPD based genetic diversity of this experiment. The software AlphaEaseFC 4.0 was used for measuring molecular weight of bands. The proportion of polymorphic loci was calculated by the equation:

$$
P=\frac{n_{p j}}{n_{\text {total }}}
$$

where, ( $P=$ proportion of polymorphic loci, $n_{p j}$ number of polymorphic loci and $n_{\text {total }}=$ total number of loci). Average number of alleles per locus was calculated by the following formula:

$$
N=\frac{1}{k} \sum_{i=o}^{k} n_{i}
$$

Here, $k$ is the number of loci and $n_{i}$ is the number of alleles detected per locus. Genetic distance analysis by using pair wise similarity was calculated by the formula:

$$
D=1-\frac{N_{x y}}{N_{x}+N_{y}-N_{x y}}
$$

where ( $D=$ genetic distance between sample $x$ and $y, N_{x y}=$ number of bands shared by sample $x$ and $y, N_{x}=$ number of bands in sample $x$ and $N_{y}=$ number of bands in sample $y$ ). Using the equation,

$$
F=\frac{2 N_{x y}}{N_{x}+N_{y}}
$$

where ( $F=$ Nei's genetic similarity, $N_{x y}$ number of shared bands between $x$ and $y, N_{x}=$ number of bands in $x$ and $N_{y}$ is the number of bands in $y$ ), Nei's genetic similarity was calculated. Linkage distance was calculated with squared Euclidean distances using the software new.sta and genetic relationships among individuals were observed based on linkage distance using the software "Statistica". 


\section{PCR amplification for glutamine synthetase gene}

Genetic diversity of the freshwater mud eel, M. cuchia was also analyzed using the Restriction Fragment Length Polymorphism (RFLP) method. A 541 bp long partial sequence of glutamine synthetase gene was allowed with two restriction enzymes. Gene specific primer of glutamine synthetase, accession no. GSase 152041 (5'-GAGGGCTCCAACAGCGATATGTA-3') and accession no. GSase 152042 (5'-CTGAAGTTTGTATGGCAGCCAGC-3') [34] were used for PCR amplification for the RFLP assay. The PCR amplification was carried out in a total volume of $15 \mu \mathrm{l}$ containing $8 \mu \mathrm{l}$ of master mix (Promega Hot Start), $1 \mu \mathrm{l}$ of primers, $2 \mu \mathrm{l}$ of template DNA and $4 \mu \mathrm{l}$ deionized distilled water. The protocol for the process was as follows: preheating for 3 minutes at $94^{\circ} \mathrm{C}$ followed by denaturation at $94^{\circ} \mathrm{C}$ for 1 minute, annealing at $64^{\circ} \mathrm{C}$ for 1 minute and 2 minutes for elongation at $72^{\circ} \mathrm{C}$ and final step at $72^{\circ} \mathrm{C}$ for 7 minutes to allow complete extension of the amplified fragments. The efficiency of the PCR products of glutamine synthetase gene of $M$. cuchia was checked by agarose-gel electrophoresis with $1.2 \%$ agarose. $1 \mathrm{~kb}$ plus DNA ladder (GeneRuler ${ }^{\mathrm{TM}}$ ) was used for checking the length of the DNA fragments at $541 \mathrm{bp}$ length. The electrophoresis was run at $75 \mathrm{~V}$ for 40 minutes. The gel was then placed in gel documentation system and photograph was taken by digital camera (Panasonic DMC-fs20). After getting good bands of GS gene compare with ladders where ladder range was started from 250 bp lengths the PCR DNA products were kept in freezer at $-20^{\circ} \mathrm{C}$ for further analysis by restriction enzymes.

\section{PCR-RFLP analysis}

The glutamine synthetase gene had an approximate size of 541 $\mathrm{bp}$ and it was digested with two restriction enzymes (+Hpy178III [TCNNGA] and +CfrI [YGGCCR]) targeting a single cleavage site to detect polymorphism of freshwater mud eel in Bangladesh. Enzymes were identified using glutamine synthetase gene base pairs through blasting and the restriction site of the enzyme was searched out from the EBI nucleotide data base. The RFLP mix was prepared using milli-Q water, enzyme and enzyme's buffer and the mixture was run through PCR products at $37^{\circ} \mathrm{C}$ for 2 hours for the enzyme $+\mathrm{Hpy}_{178 \mathrm{III}}{ }^{*}$ and at $55^{\circ} \mathrm{C}$ for two hours and $80^{\circ} \mathrm{C}$ for 20 minutes for the enzyme $+\mathrm{CfrI}$. The efficiency of the PCR-RFLP products of freshwater mud eel was checked by $1.5 \%$ agarose at $100 \mathrm{~V}$ for 60 minutes. An image of the DNA fragments was taken by a digital camera through gel documentation system and the length of the restriction fragments was checked by comparing with the $1 \mathrm{~kb}$ plus DNA ladder (GeneRuler ${ }^{\mathrm{TM}}$ ) while the ladder was started from $250 \mathrm{bp}$ length.

\section{Calculating the proportion of shared fragments and genetic distances}

The restriction patterns of individuals of freshwater mud eel were compared to detect polymorphism, using matrices based on either presence or absence or relative abundance of bands. The similarity index or proportion of shared fragments $(F)$ was calculated by comparing banding patterns between the two individuals. The formula used for this calculation is:

$$
F=\frac{2 N_{x y}}{N_{x}+N_{y}}
$$

( $F=$ Nei's genetic similarity, $N_{x y}=$ number of shared bands between $x$ and $y, N_{x}=$ number of bands in $x$ and $N_{y}$ is the number of bands in $y$ ). A matrix of genetic distances (p-value) between all individuals based on dissimilarity indices were calculated and the formula used for this calculation is:

$$
P=1-\left[\frac{\left\{\left(\mathrm{F}^{2}+8 \mathrm{~F}\right)^{\frac{1}{2}}-\mathrm{F}\right\}}{2}\right]^{\frac{1}{r}}
$$

Here, $r$ is the average number of enzyme sequences. Both calculations were done by using Microsoft Office Excel 2016.

\section{Results}

\section{RAPD based genetic diversity}

DNA profiling and data scoring: DNA profiling and data scoring were studied separately for each primer. Each amplified banding profile was defined by the presence (1) or absence (0) of bands at particular positions. The bands of different primers were seen in different levels of length of DNA. A total of 735 bands with 228 polymorphic loci were detected among the selected 30 individuals (Table 1). Polymorphic loci were revealed by all the primers with $100 \%$ polymorphism. The highest number of bands (120) was amplified by the primer C-04, while UBC122 produced the lowest. The highest number of bands (4.00) per individual was amplified from the primer C-04 and the lowest number of bands (1.63) per individual was amplified by the primer UBC122.

\section{Genetic distances}

Inter individual pair-wise similarity of $M$. cuchia was studied and based on the similarity data, 13 diverse groups of individuals $(14,12$, $11,10,9,8,7,6,5,4,3,2$ and 1 ) were found. The genetic distance among individuals of $M$. cuchia was calculated by using the data from pair-wise similarity index where 36 groups of genetic diversities were measured. The lowest genetic distance was found between the individuals 7 and $16(0.58)$ followed by 7 and $11(0.59)$ and 11 and $20(0.60)$, and the highest genetic distance was recorded 0.97 between the individual pairs of 17 and 22, 14 and 24 and 10 and 24 and second highest was found 0.96 between the individual pairs of 6 and 24 and 5 and 24 respectively. Relatively higher genetic distance was recorded in other individuals (Table 2). The average genetic diversity was found 0.8173716 and which indicates the good genetic status of this experimental fish in Bangladeshi nature especially in the experimental ecosystem. Considering the Nei's genetic similarity analysis, different values of similarities ranges from 0.06 to 0.6 were found by these 30 individuals where average value was recorded 0.301746 which was very relevant to genetic distance.

\section{Genetic relationships among individuals}

Based on Squared Euclidean Distances, the values of pair-wise comparisons of the linkage were computed from combined data of these experimental individuals. Different levels of values of linkage distance were found which was ranged from 3.6 to 6.24, and based on different linkage distances, cluster analysis using Unweighted Pair Group Method with Arithmetic Mean (UPGMA) was done to resolve the phylogenetic relationships among experimental individuals of $M$. cuchia (Figure 1). The UPGMA clustering system generated six clusters by 11 clades where 22 individuals/samples were involved to form 11 clades and the rest of the samples were connected to those clades with a specific linkage distance. Sample (S) 1 and Sample $4(=S 4)$ were found closely related as clade 1 which was created cluster 1 with clade 2 (S5, S6). Both of these clades were situated between the linkage distances of 3.5 to 4 . Sample S3, S2, S13, S10 and S15 were individually connected to the clade 1 and clade 2 . Although they were somehow distantly related, but situated under the cluster 1 , however, their linkage distance was observed approximately 4.9. Clade 3 (S7, S16) and clade 4 (S11, S20) were close enough between the linkage distance of 4-4.5 and formed 
Citation: Md. Miah F, Anwar S, Md. Ali H, Naser MN, Ahmed K (2018) Assessment of Genetic Diversity of Freshwater Mud Eel (Monopterus cuchia) Using RAPD and RFLP Markers. J Aquac Res Development 9: 543. doi: 10.4172/2155-9546.1000543

Page 4 of 8

\begin{tabular}{|c|c|c|c|c|c|c|}
\hline Primers & $\begin{array}{l}\text { Size of DNA } \\
\text { (bp) }\end{array}$ & Total no. of Bands & $\begin{array}{l}\text { Polymorphic loci } \\
\text { (No.) }\end{array}$ & $\begin{array}{l}\text { Monomorphi loci } \\
\text { (No.) }\end{array}$ & $\begin{array}{l}\text { Polymorphic loci } \\
(\%)\end{array}$ & $\begin{array}{l}\text { Avg. no. of Bands } \\
\text { per sample }\end{array}$ \\
\hline В O3 & $151-2608$ & 88 & 36 & 00 & $100 \%$ & 2.93 \\
\hline OPF 14 & $71-1566$ & 107 & 24 & 00 & $100 \%$ & 3.57 \\
\hline $\mathrm{C} \mathrm{O} 4$ & $40-1473$ & 120 & 26 & 00 & $100 \%$ & 4.00 \\
\hline OPB 05 & $71-1605$ & 105 & 33 & 00 & $100 \%$ & 3.50 \\
\hline OPB 08 & $60-1605$ & 100 & 23 & 00 & $100 \%$ & 3.33 \\
\hline OPB 19 & $170-1920$ & 109 & 42 & 00 & $100 \%$ & 3.63 \\
\hline OPB 12 & $280-2703$ & 57 & 32 & 00 & $100 \%$ & 1.90 \\
\hline UBC122 & $310-635$ & 49 & 12 & 00 & $100 \%$ & 1.63 \\
\hline Total & -- & 735 & 228 & 00 & -- & -- \\
\hline Average & -- & 91.88 & -- & -- & -- & -- \\
\hline
\end{tabular}

Table 1: Summary of the bands revealed from eight RAPD primers.

\begin{tabular}{|c|c|c|c|c|c|c|c|c|c|c|c|c|c|c|c|c|c|c|c|c|c|c|c|c|c|c|c|c|c|c|}
\hline & - & $\sim$ & $m$ & $\nabla$ & เ & 0 & $\wedge$ & $\infty$ & $\sigma$ & $\stackrel{\circ}{\circ}$ & $F$ & $\simeq$ & $\stackrel{m}{\stackrel{m}{2}}$ & $\stackrel{\nabla}{\leftarrow}$ & $\stackrel{\circ}{\leftarrow}$ & $\stackrel{\circ}{\circ}$ & 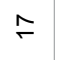 & 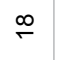 & $\stackrel{\mathscr{O}}{\leftarrow}$ & กิ & $\bar{N}$ & $\approx$ & $\widetilde{N}$ & $\stackrel{\Xi}{N}$ & 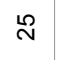 & $\stackrel{\circ}{\sim}$ & $\hat{N}$ & $\stackrel{\infty}{\sim}$ & $\stackrel{\sim}{N}$ & ஓ \\
\hline- & 0 & $\begin{array}{l}\infty \\
\infty \\
0 \\
0\end{array}$ & $\begin{array}{c}\infty \\
\stackrel{\infty}{\infty} \\
0\end{array}$ & $\begin{array}{l}\hat{0} \\
0 \\
0\end{array}$ & $\begin{array}{l}\infty \\
\infty \\
0\end{array}$ & 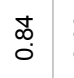 & $\begin{array}{c}\infty \\
\infty \\
\infty \\
0\end{array}$ & চ. & @̊ & @̊ & \begin{tabular}{l|l}
$\infty$ \\
$\infty$ \\
0 \\
0
\end{tabular} & $\underset{\infty}{\infty}$ & $\begin{array}{c}\infty \\
\stackrel{\infty}{\infty} \\
0\end{array}$ & $\begin{array}{l}\hat{\infty} \\
0 \\
0\end{array}$ & 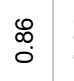 & 离 & $\begin{array}{c}\cong \\
\infty \\
0\end{array}$ & $\begin{array}{c}\bar{\infty} \\
0 \\
0\end{array}$ & 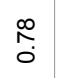 & $\begin{array}{l}\hat{N} \\
0\end{array}$ & $\begin{array}{l}R \\
0 \\
0\end{array}$ & $\begin{array}{c}\mathscr{8} \\
\stackrel{8}{\circ}\end{array}$ & $\begin{array}{l}\text { s. } \\
0\end{array}$ & $\begin{array}{c}\hat{c} \\
\text { co } \\
0\end{array}$ & $\begin{array}{c}\infty \\
\infty \\
0\end{array}$ & $\begin{array}{l}\bar{\infty} \\
0 \\
0\end{array}$ & $\begin{array}{c}\dot{c} \\
\text { o } \\
0\end{array}$ & $\begin{array}{c}\infty \\
\infty \\
0\end{array}$ & $\begin{array}{l}\infty \\
\infty \\
0\end{array}$ & 离 \\
\hline$\sim$ & & 0 & $\begin{array}{l}\text { t } \\
\hat{O}\end{array}$ & $\begin{array}{l}\stackrel{2}{2} \\
0\end{array}$ & $\begin{array}{c}\hat{o} \\
\text { o. } \\
0\end{array}$ & $\begin{array}{l}0 \\
\infty \\
0\end{array}$ & 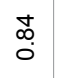 & 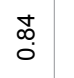 & $\begin{array}{l}\infty \\
\infty \\
0 \\
0\end{array}$ & $\begin{array}{l}\stackrel{\infty}{\infty} \\
\infty \\
0\end{array}$ & 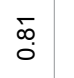 & ㅇ. & $\begin{array}{l}\stackrel{R}{0} \\
0\end{array}$ & ֻ̊. & ঃ̊. & 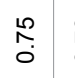 & $\begin{array}{l}\stackrel{2}{2} \\
0\end{array}$ & $\begin{array}{l}N \\
0 \\
0\end{array}$ & $\begin{array}{l}\infty \\
\infty \\
\infty \\
0\end{array}$ & $\begin{array}{l}\infty \\
\infty \\
0 \\
0\end{array}$ & $\begin{array}{c}\hat{N} \\
0\end{array}$ & 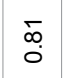 & $\begin{array}{c}\infty \\
\infty \\
\infty \\
0\end{array}$ & $\begin{array}{l}\stackrel{8}{\infty} \\
0 \\
0\end{array}$ & $\begin{array}{l}\text { d } \\
\text { o } \\
0\end{array}$ & $\begin{array}{l}\text { d } \\
\text { o } \\
0\end{array}$ & $\begin{array}{l}\infty \\
\infty \\
0\end{array}$ & $\begin{array}{c}\infty \\
\infty \\
0\end{array}$ & $\begin{array}{l}\text { d } \\
\text { o } \\
0\end{array}$ & \\
\hline$m$ & & & 。 & $\begin{array}{l}R \\
0 \\
0\end{array}$ & $\begin{array}{l}\bar{\infty} \\
0 \\
0\end{array}$ & $\begin{array}{l}\stackrel{0}{2} \\
0\end{array}$ & $\underset{0}{2}$ & $\underset{\infty}{\infty}$ & $\begin{array}{l}\infty \\
\infty \\
0 \\
0\end{array}$ & $\begin{array}{l}\Delta \\
\text { o } \\
0\end{array}$ & $\begin{array}{l}\infty \\
\infty \\
0\end{array}$ & $\begin{array}{l}\infty \\
\infty \\
0\end{array}$ & $\begin{array}{c}\infty \\
\infty \\
0\end{array}$ & $\begin{array}{l}\bar{\delta} \\
0\end{array}$ & $\begin{array}{l}\infty \\
\infty \\
0\end{array}$ & $\begin{array}{l}R \\
0 \\
0\end{array}$ & $\begin{array}{c}\infty \\
\infty \\
0\end{array}$ & 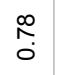 & $\begin{array}{l}R \\
R \\
0\end{array}$ & $\begin{array}{l}\infty \\
\infty \\
0\end{array}$ & $\begin{array}{l}\bar{\infty} \\
0 \\
0\end{array}$ & $\begin{array}{c}\infty \\
\infty \\
0 \\
0\end{array}$ & $\begin{array}{l}\infty \\
\infty \\
0 \\
0\end{array}$ & $\begin{array}{c}\infty \\
\infty \\
0\end{array}$ & $\begin{array}{c}\stackrel{0}{\infty} \\
0\end{array}$ & 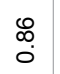 & $\begin{array}{c}\bar{\infty} \\
0 \\
0\end{array}$ & $\begin{array}{l}\hat{N} \\
0\end{array}$ & $\begin{array}{c}\stackrel{0}{0} \\
\stackrel{0}{0}\end{array}$ & 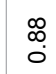 \\
\hline$\sigma$ & & & & 0 & 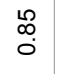 & $\stackrel{+}{\stackrel{N}{0}}$ & $\begin{array}{l}\mathscr{\infty} \\
\infty \\
0\end{array}$ & $\begin{array}{c}\bar{\infty} \\
0 \\
0\end{array}$ & $\begin{array}{l}\infty \\
\infty \\
\infty \\
0\end{array}$ & $\begin{array}{c}\hat{c} \\
\stackrel{0}{0} \\
0\end{array}$ & $\begin{array}{l}\stackrel{\infty}{\infty} \\
\infty \\
0\end{array}$ & $\begin{array}{l}2 \\
\stackrel{2}{0}\end{array}$ & $\begin{array}{l}8 \\
\circ \\
0\end{array}$ & $\begin{array}{l}\infty \\
\infty \\
\infty \\
0\end{array}$ & $\begin{array}{l}0 \\
\infty \\
0\end{array}$ & $\begin{array}{l}\infty \\
\stackrel{0}{0} \\
0\end{array}$ & $\begin{array}{l}\stackrel{8}{\circ} \\
\circ\end{array}$ & $\begin{array}{l}\hat{A} \\
0\end{array}$ & 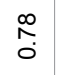 & $\begin{array}{l}0 \\
\infty \\
0\end{array}$ & $\begin{array}{l}R \\
0 \\
0\end{array}$ & $\begin{array}{l}\mathscr{D} \\
\infty \\
0\end{array}$ & $\begin{array}{l}\infty \\
\infty \\
0 \\
0\end{array}$ & $\begin{array}{c}0 \\
\infty \\
0\end{array}$ & $\begin{array}{l}\bar{\infty} \\
0 \\
0\end{array}$ & $\begin{array}{l}\bar{\infty} \\
0 \\
0\end{array}$ & $\begin{array}{l}\infty \\
\infty \\
0\end{array}$ & $\begin{array}{l}\overrightarrow{0} \\
\infty \\
0\end{array}$ & $\begin{array}{l}\infty \\
\infty \\
0 \\
0\end{array}$ & $\begin{array}{l}\infty \\
0 \\
0\end{array}$ \\
\hline$\infty$ & & & & & 0 & 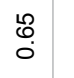 & $\begin{array}{l}\stackrel{R}{2} \\
0 \\
0\end{array}$ & $\begin{array}{l}\infty \\
\infty \\
0\end{array}$ & $\begin{array}{l}\infty \\
\substack{\infty \\
0}\end{array}$ & 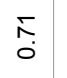 & $\begin{array}{c}\stackrel{0}{\infty} \\
0 \\
0\end{array}$ & $\begin{array}{l}\stackrel{\infty}{\infty} \\
0 \\
0\end{array}$ & 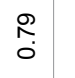 & 总 & $\begin{array}{c}\infty \\
\infty \\
0 \\
0\end{array}$ & $\begin{array}{c}\stackrel{0}{\infty} \\
\infty \\
0\end{array}$ & $\begin{array}{l}\bar{\delta} \\
0 \\
0\end{array}$ & $\begin{array}{l}\stackrel{8}{\infty} \\
0 \\
0\end{array}$ & $\begin{array}{l}\stackrel{R}{2} \\
0\end{array}$ & $\begin{array}{l}\infty \\
\infty \\
0 \\
0\end{array}$ & $\begin{array}{l}\stackrel{+}{\infty} \\
\infty \\
0\end{array}$ & $\begin{array}{c}\text { J } \\
\text { o }\end{array}$ & $\begin{array}{l}\infty \\
\infty \\
0\end{array}$ & $\begin{array}{l}8 \\
8 \\
0\end{array}$ & $\begin{array}{l}\stackrel{\infty}{\infty} \\
0 \\
0\end{array}$ & $\begin{array}{l}\text { मे } \\
\stackrel{\circ}{\circ}\end{array}$ & $\begin{array}{l}\stackrel{\infty}{\infty} \\
0 \\
0\end{array}$ & $\begin{array}{l}\infty \\
\infty \\
0\end{array}$ & $\begin{array}{l}\hat{o} \\
0 \\
0\end{array}$ & চু \\
\hline 0 & & & & & & $\circ$ & $\begin{array}{l}R \\
0 \\
0\end{array}$ & 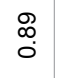 & $\begin{array}{l}\stackrel{2}{2} \\
\stackrel{0}{0}\end{array}$ & $\begin{array}{l}\stackrel{R}{\stackrel{2}{0}} \\
0\end{array}$ & $\stackrel{\infty}{\stackrel{\infty}{0}}$ & $\begin{array}{l}\stackrel{0}{2} \\
\stackrel{0}{0}\end{array}$ & $\stackrel{8}{\circ}$ & $\begin{array}{l}\tilde{\hat{O}} \\
\mathbf{0}\end{array}$ & $\begin{array}{l}\stackrel{R}{2} \\
\stackrel{0}{0}\end{array}$ & $\begin{array}{l}8 \\
0 \\
0\end{array}$ & $\begin{array}{l}\circ \\
\stackrel{\circ}{\circ}\end{array}$ & $\begin{array}{l}\stackrel{t}{\circ} \\
\stackrel{\circ}{\circ}\end{array}$ & $\begin{array}{l}\infty \\
\infty \\
\infty \\
0\end{array}$ & $\begin{array}{l}0 \\
\infty \\
0 \\
0\end{array}$ & 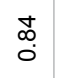 & @ & $\begin{array}{l}0 \\
\stackrel{2}{0}\end{array}$ & $\begin{array}{l}\stackrel{8}{\circ} \\
\circ\end{array}$ & $\begin{array}{l}n \\
\stackrel{0}{0} \\
0\end{array}$ & $\begin{array}{l}0 \\
\infty \\
0\end{array}$ & $\begin{array}{l}\bar{\infty} \\
0 \\
0\end{array}$ & 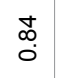 & $\begin{array}{l}\circ \\
\stackrel{\circ}{\circ}\end{array}$ & o \\
\hline$\wedge$ & & & & & & & 0 & $\hat{0}$ & 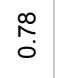 & $\underset{⿱}{\stackrel{N}{0}}$ & 号 & 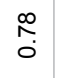 & 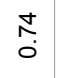 & \begin{tabular}{l}
$\stackrel{\infty}{\infty}$ \\
\hdashline \\
0
\end{tabular} & $\hat{i}$ & 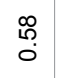 & 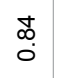 & 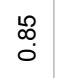 & $\begin{array}{l}\mathbb{d} \\
\infty \\
0 \\
0\end{array}$ & $\begin{array}{l}8 \\
0 \\
0\end{array}$ & $\begin{array}{l}\infty \\
\infty \\
\infty \\
0\end{array}$ & $\begin{array}{c}\infty \\
\infty \\
0 \\
0\end{array}$ & $\begin{array}{l}\infty \\
\infty \\
\infty \\
0\end{array}$ & $\begin{array}{l}\infty \\
\infty \\
\infty \\
0\end{array}$ & $\begin{array}{l}\stackrel{0}{2} \\
\stackrel{0}{0}\end{array}$ & 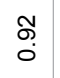 & $\begin{array}{l}0 \\
\stackrel{2}{0} \\
0\end{array}$ & $\begin{array}{l}\stackrel{N}{\infty} \\
\underset{0}{0}\end{array}$ & 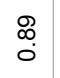 & ه্. \\
\hline$\infty$ & & & & & & & & 0 & 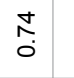 & 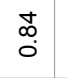 & $\begin{array}{l}R \\
\stackrel{R}{0}\end{array}$ & $\begin{array}{l}N \\
\hat{O}\end{array}$ & 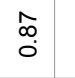 & 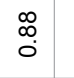 & $\begin{array}{l}\vec{d} \\
\infty \\
0 \\
0\end{array}$ & $\begin{array}{l}\stackrel{0}{2} \\
\stackrel{0}{0}\end{array}$ & $\begin{array}{l}\hat{N} \\
0\end{array}$ & $\begin{array}{l}\stackrel{\infty}{\infty} \\
\infty \\
0\end{array}$ & $\begin{array}{l}0 \\
\stackrel{0}{0} \\
0\end{array}$ & $\begin{array}{l}0 \\
\infty \\
0\end{array}$ & $\begin{array}{l}\mathscr{m} \\
\stackrel{0}{0} \\
0\end{array}$ & $\begin{array}{l}\infty \\
\substack{\infty \\
0 \\
0}\end{array}$ & $\begin{array}{l}0 \\
\stackrel{0}{0} \\
0\end{array}$ & 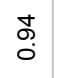 & $\begin{array}{l}\infty \\
\infty \\
0 \\
0\end{array}$ & 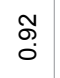 & $\begin{array}{l}\stackrel{\infty}{\infty} \\
\infty \\
0\end{array}$ & $\begin{array}{l}\stackrel{2}{2} \\
0 \\
0\end{array}$ & 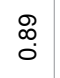 & $\begin{array}{l}\infty \\
\infty \\
\infty \\
0\end{array}$ \\
\hline 0 & & & & & & & & & 。 & $\begin{array}{l}\stackrel{R}{R} \\
0 \\
0\end{array}$ & $\hat{0}$ & $\begin{array}{l}\stackrel{R}{2} \\
\stackrel{0}{0}\end{array}$ & $\begin{array}{c}\stackrel{0}{\infty} \\
\stackrel{0}{0}\end{array}$ & 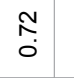 & $\stackrel{\infty}{\stackrel{\infty}{0}}$ & 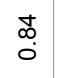 & $\begin{array}{l}\stackrel{\infty}{\infty} \\
0 \\
0\end{array}$ & $\begin{array}{l}8 \\
\circ \\
0\end{array}$ & $\begin{array}{l}\stackrel{0}{\infty} \\
0 \\
0\end{array}$ & ঠ். & $\begin{array}{l}0 \\
\infty \\
0\end{array}$ & $\begin{array}{c}\bar{\infty} \\
0 \\
0\end{array}$ & $\begin{array}{l}\mathbb{\infty} \\
\stackrel{\infty}{\infty} \\
0\end{array}$ & 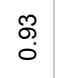 & $\begin{array}{l}\mathbb{d} \\
\stackrel{0}{0} \\
0\end{array}$ & ঠ். & $\begin{array}{l}\mathbb{\infty} \\
\stackrel{\infty}{\infty} \\
0\end{array}$ & $\begin{array}{l}\mathbb{\infty} \\
\stackrel{\infty}{0} \\
0\end{array}$ & $\begin{array}{l}\mathbb{d} \\
\stackrel{0}{0} \\
0\end{array}$ & \begin{tabular}{c}
$\infty$ \\
\hdashline \\
\hdashline
\end{tabular} \\
\hline$\stackrel{\circ}{\circ}$ & & & & & & & & & & 0 & $\hat{0}$ & $\begin{array}{l}\stackrel{R}{2} \\
\stackrel{0}{0}\end{array}$ & $\begin{array}{l}R \\
\stackrel{R}{0}\end{array}$ & 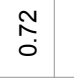 & $\begin{array}{l}R \\
\text { R }\end{array}$ & $\begin{array}{l}8 \\
\circ \\
\end{array}$ & $\tilde{\delta}$ & 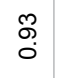 & $\stackrel{\infty}{\stackrel{\infty}{\circ}}$ & 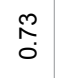 & $\begin{array}{l}0 \\
\infty \\
0\end{array}$ & $\begin{array}{l}\text { To } \\
0 \\
0\end{array}$ & $\begin{array}{l}0 \\
\infty \\
0\end{array}$ & ồ. & 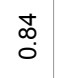 & ঠ் & $\begin{array}{l}\stackrel{8}{\&} \\
\stackrel{8}{0}\end{array}$ & $\begin{array}{l}\stackrel{8}{\&} \\
\stackrel{8}{0}\end{array}$ & 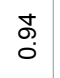 & 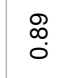 \\
\hline$F$ & & & & & & & & & & & 0 & $\begin{array}{l}\stackrel{\infty}{\stackrel{0}{0}} \\
\dot{0}\end{array}$ & $\begin{array}{l}\bar{\infty} \\
\dot{0} \\
0\end{array}$ & $\begin{array}{l}\infty \\
\infty \\
0 \\
0\end{array}$ & $\begin{array}{l}R \\
\stackrel{R}{0}\end{array}$ & $\begin{array}{l}\stackrel{0}{2} \\
\stackrel{0}{0}\end{array}$ & $\begin{array}{l}\vec{\Phi} \\
0 \\
0\end{array}$ & $\begin{array}{l}\stackrel{\infty}{\infty} \\
0 \\
0\end{array}$ & $\begin{array}{l}\mathbf{d} \\
\stackrel{0}{0} \\
0\end{array}$ & $\stackrel{8}{0}$ & $\stackrel{8}{\circ}$ & $\begin{array}{l}\infty \\
\stackrel{0}{0} \\
0\end{array}$ & $\begin{array}{l}\stackrel{\infty}{\infty} \\
0 \\
0\end{array}$ & $\begin{array}{l}\text { d } \\
\stackrel{\leftrightarrow}{0}\end{array}$ & 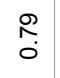 & 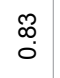 & $\begin{array}{l}\stackrel{R}{2} \\
\stackrel{0}{0}\end{array}$ & $\begin{array}{l}\stackrel{\infty}{\infty} \\
0 \\
0\end{array}$ & $\begin{array}{l}0 \\
\infty \\
0\end{array}$ & $\stackrel{\substack{\infty \\
\hdashline}}{\infty}$ \\
\hline$\cong$ & & & & & & & & & & & & 0 & 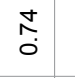 & $\begin{array}{l}\stackrel{\mathscr{L}}{\circ} \\
\stackrel{0}{0}\end{array}$ & $\begin{array}{l}\hat{\hat{O}} \\
\hat{0}\end{array}$ & 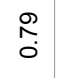 & 옹 & $\begin{array}{l}\stackrel{\infty}{\infty} \\
\infty \\
0\end{array}$ & $\underset{\substack{t \\
0 \\
0}}{0}$ & $\begin{array}{l}\infty \\
\infty \\
0 \\
0\end{array}$ & \&: & $\begin{array}{l}\infty \\
\infty \\
0\end{array}$ & 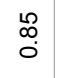 & $\begin{array}{l}\text { d. } \\
\text { o }\end{array}$ & 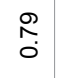 & 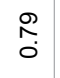 & $\begin{array}{l}\stackrel{0}{\infty} \\
\stackrel{\infty}{0}\end{array}$ & 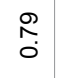 & $\begin{array}{l}0 \\
\infty \\
0\end{array}$ & $\stackrel{\substack{\infty \\
\infty}}{\infty}$ \\
\hline$\stackrel{m}{\sim}$ & & & & & & & & & & & & & 0 & $\begin{array}{c}\mathscr{D} \\
0 \\
0\end{array}$ & $\begin{array}{l}\hat{1} \\
0\end{array}$ & $\begin{array}{l}\infty \\
\stackrel{\infty}{0} \\
0\end{array}$ & $\begin{array}{l}\hat{N} \\
0\end{array}$ & 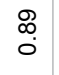 & $\begin{array}{l}\bar{\infty} \\
\vdots \\
0\end{array}$ & $\begin{array}{l}\bar{\infty} \\
\vdots \\
0\end{array}$ & $\begin{array}{l}\infty \\
œ \\
0\end{array}$ & $\begin{array}{l}\mathbb{D} \\
\stackrel{5}{0} \\
0\end{array}$ & 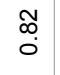 & 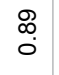 & $\begin{array}{c}0 \\
\infty \\
0\end{array}$ & 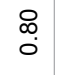 & $\begin{array}{l}0 \\
\stackrel{0}{0}\end{array}$ & $\begin{array}{l}\stackrel{0}{0} \\
\stackrel{0}{0}\end{array}$ & 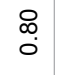 & $\begin{array}{l}\stackrel{R}{0} \\
\text { o. }\end{array}$ \\
\hline$\underset{\leftarrow}{\ddagger}$ & & & & & & & & & & & & & & 0 & $\begin{array}{l}\widetilde{N} \\
\stackrel{\infty}{0} \\
0\end{array}$ & 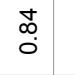 & 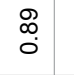 & $\begin{array}{l}\mathscr{\infty} \\
\stackrel{0}{0}\end{array}$ & 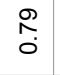 & 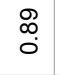 & $\begin{array}{l}\infty \\
\infty \\
0 \\
0\end{array}$ & $\begin{array}{l}\stackrel{t}{\circ} \\
\stackrel{\circ}{0}\end{array}$ & $\begin{array}{l}0 \\
\substack{\infty \\
0}\end{array}$ & $\begin{array}{c}\hat{D} \\
\text { ò }\end{array}$ & $\begin{array}{c}\bar{\lambda} \\
0\end{array}$ & 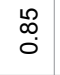 & 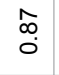 & $\begin{array}{l}\text { \& } \\
\text { O }\end{array}$ & ঠু & ه্. \\
\hline$\stackrel{\circ}{\leftarrow}$ & & & & & & & & & & & & & & & 0 & $\begin{array}{l}0 \\
0 \\
0 \\
0\end{array}$ & $\begin{array}{l}\infty \\
\infty \\
0 \\
0\end{array}$ & $\begin{array}{l}\tilde{\delta} \\
\text { Oे }\end{array}$ & $\begin{array}{l}\bar{\infty} \\
0 \\
0\end{array}$ & $\begin{array}{l}\bar{\infty} \\
0 \\
0\end{array}$ & $\begin{array}{l}\infty \\
\infty \\
0\end{array}$ & $\begin{array}{c}\infty \\
\infty \\
0 \\
0\end{array}$ & 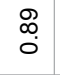 & $\begin{array}{l}\mathscr{8} \\
\stackrel{0}{0}\end{array}$ & 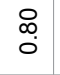 & $\stackrel{\delta}{\delta}$ & $\begin{array}{l}\infty \\
\infty \\
0 \\
0\end{array}$ & 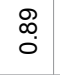 & $\begin{array}{l}\text { J } \\
\text { o }\end{array}$ & $œ$ \\
\hline$\stackrel{\circ}{\circ}$ & & & & & & & & & & & & & & & & 0 & $\begin{array}{l}\infty \\
\infty \\
0 \\
0\end{array}$ & $\begin{array}{l}0 \\
\stackrel{0}{0}\end{array}$ & $\begin{array}{l}0 \\
\infty \\
0 \\
0\end{array}$ & $\underset{\mathfrak{N}}{N}$ & 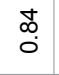 & $\begin{array}{l}\mathbb{\infty} \\
\stackrel{\infty}{0} \\
0\end{array}$ & $\begin{array}{l}\bar{\infty} \\
0 \\
0\end{array}$ & ঠু & $\stackrel{N}{N}$ & $\begin{array}{l}\mathscr{D} \\
\stackrel{\infty}{0} \\
0\end{array}$ & $\begin{array}{l}\stackrel{R}{\stackrel{2}{0}} \\
\stackrel{0}{0}\end{array}$ & 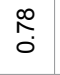 & $\begin{array}{l}\tilde{\delta} \\
\delta\end{array}$ & 赵 \\
\hline $\mathcal{F}$ & & & & & & & & & & & & & & & & & 0 & 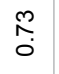 & $\begin{array}{l}\hat{i} \\
0\end{array}$ & $\stackrel{\substack{\infty \\
0}}{0}$ & $\stackrel{\mathscr{O}}{\circ}$ & 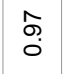 & $\begin{array}{l}\stackrel{8}{\mathscr{O}} \\
\stackrel{0}{0}\end{array}$ & $\begin{array}{l}\stackrel{\infty}{\infty} \\
0 \\
0\end{array}$ & $\begin{array}{l}\infty \\
\stackrel{0}{0}\end{array}$ & $\begin{array}{l}\infty \\
\substack{\infty \\
0}\end{array}$ & $\begin{array}{l}\stackrel{0}{0} \\
\stackrel{0}{0}\end{array}$ & 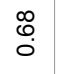 & $\begin{array}{l}\text { to } \\
0 \\
0\end{array}$ & $0^{\circ}$ \\
\hline
\end{tabular}


Citation: Md. Miah F, Anwar S, Md. Ali H, Naser MN, Ahmed K (2018) Assessment of Genetic Diversity of Freshwater Mud Eel (Monopterus cuchia) Using RAPD and RFLP Markers. J Aquac Res Development 9: 543. doi: 10.4172/2155-9546.1000543

Page 5 of 8

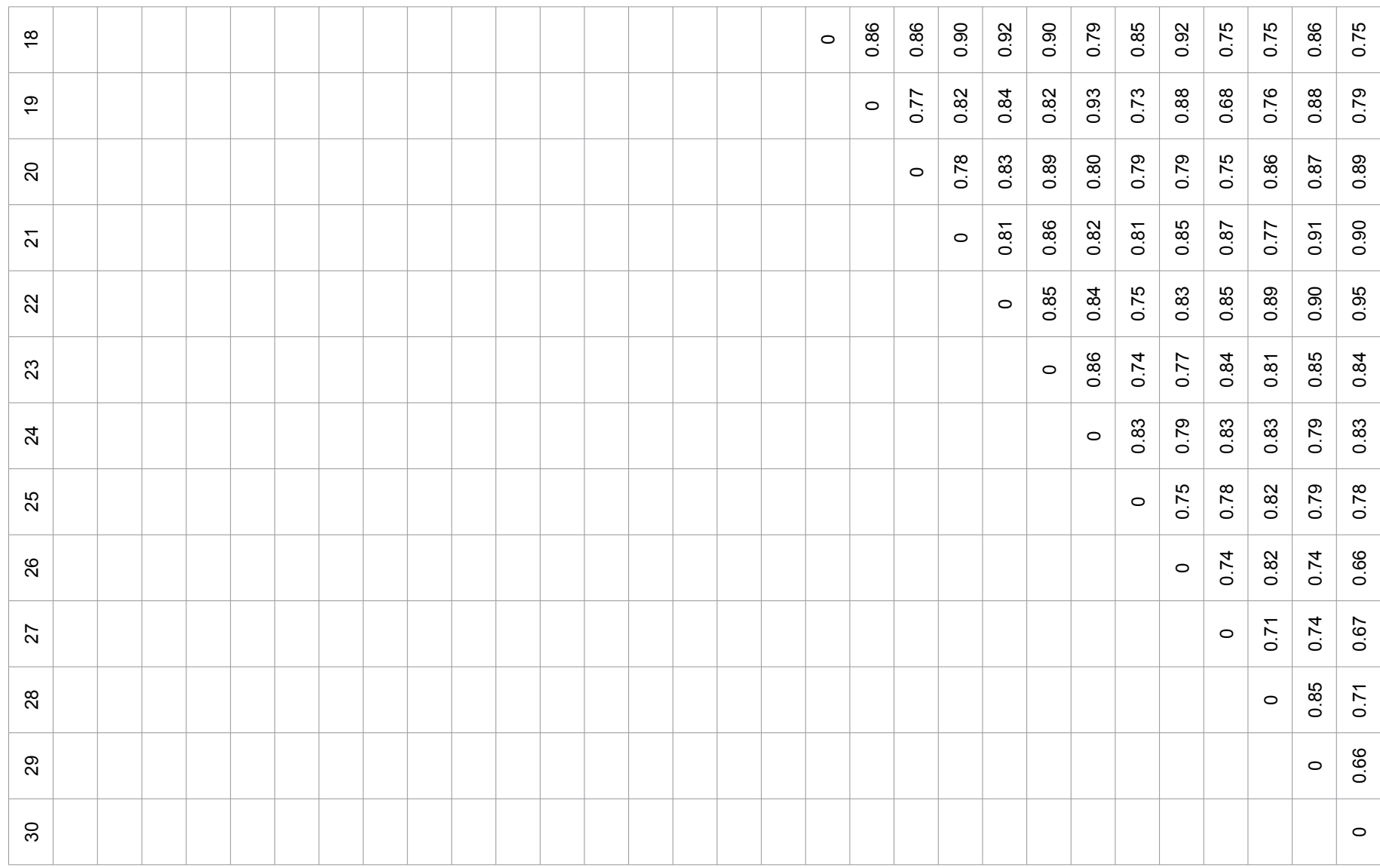

Table 2: Genetic distance among individuals of $M$. cuchia.

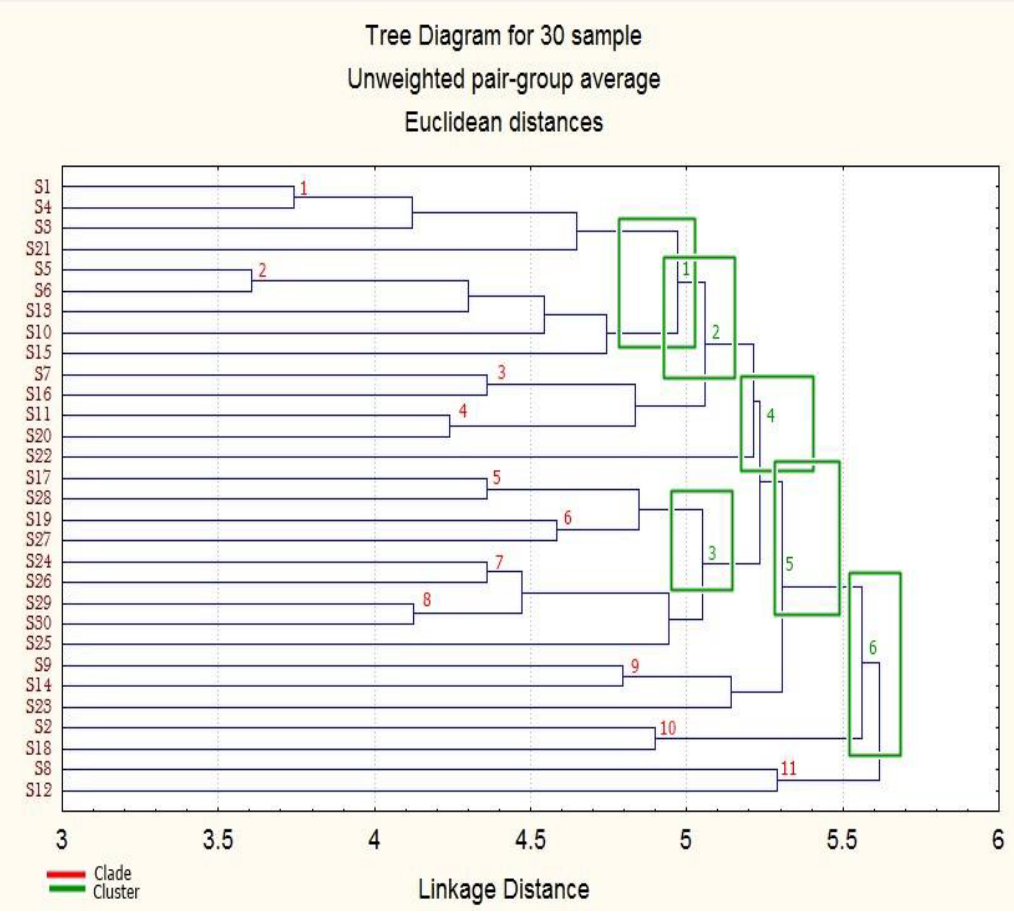

Figure 1: Relationships among the 30 individuals of $M$. cuchia. 
Citation: Md. Miah F, Anwar S, Md. Ali H, Naser MN, Ahmed K (2018) Assessment of Genetic Diversity of Freshwater Mud Eel (Monopterus cuchia) Using RAPD and RFLP Markers. J Aquac Res Development 9: 543. doi: 10.4172/2155-9546.1000543

Page 6 of 8

\begin{tabular}{|c|c|c|c|c|c|c|c|c|c|c|c|c|c|c|c|c|c|c|c|c|c|c|c|c|c|c|c|c|c|}
\hline & - & $N$ & $m$ & ナ & is & 0 & $r$ & $\infty$ & o & 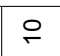 & $F$ & $\simeq$ & $\stackrel{m}{\sim}$ & $\stackrel{\nabla}{\square}$ & $\stackrel{2}{\llcorner}$ & $\stackrel{\varphi}{\circ}$ & $F$ & $\stackrel{\infty}{\sim}$ & $\stackrel{9}{\longrightarrow}$ & 尺 & $\bar{N}$ & N & $\stackrel{\infty}{\sim}$ & $d$ & $\stackrel{2}{\sim}$ & $\stackrel{2}{\sim}$ & $\hat{\sim}$ & $\stackrel{\infty}{\sim}$ & N \\
\hline 1 & & & & & & & & & & & & & & & & & & & & & & & & & & & & & \\
\hline 2 & 0 & & & & & & & & & & & & & & & & & & & & & & & & & & & & \\
\hline 3 & 0 & 0 & & & & & & & & & & & & & & & & & & & & & & & & & & & \\
\hline 4 & 0 & 0 & 0 & & & & & & & & & & & & & & & & & & & & & & & & & & \\
\hline 5 & 0 & 0 & 0 & 0 & & & & & & & & & & & & & & & & & & & & & & & & & \\
\hline 6 & 0 & 0 & 0 & 0 & 0 & & & & & & & & & & & & & & & & & & & & & & & & \\
\hline 7 & 0 & 0 & 0 & 0 & 0 & 0 & & & & & & & & & & & & & & & & & & & & & & & \\
\hline 8 & 0 & 0 & 0 & 0 & 0 & 0 & 0 & & & & & & & & & & & & & & & & & & & & & & \\
\hline 9 & 0 & 0 & 0 & 0 & 0 & 0 & 0 & 0 & & & & & & & & & & & & & & & & & & & & & \\
\hline 10 & 0 & 0 & 0 & 0 & 0 & 0 & 0 & 0 & 0 & & & & & & & & & & & & & & & & & & & & \\
\hline 11 & 0 & 0 & 0 & 0 & 0 & 0 & 0 & 0 & 0 & 0 & & & & & & & & & & & & & & & & & & & \\
\hline 12 & 0 & 0 & 0 & 0 & 0 & 0 & 0 & 0 & 0 & 0 & 0 & & & & & & & & & & & & & & & & & & \\
\hline 13 & $\begin{array}{l}\tilde{O}_{0} \\
0 \\
0 \\
0\end{array}$ & 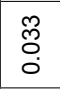 & 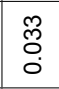 & \begin{tabular}{|l|}
$\tilde{o}$ \\
0 \\
0 \\
0
\end{tabular} & $\begin{array}{l}0 \\
\tilde{O} \\
0 \\
0\end{array}$ & $\begin{array}{l}m \\
\tilde{m} \\
0 \\
0\end{array}$ & $\begin{array}{l}\text { mo } \\
\text { Oू. } \\
0\end{array}$ & $\begin{array}{l}m \\
\tilde{m} \\
0 \\
0\end{array}$ & $\begin{array}{l}0 \\
\tilde{O} \\
0 \\
0\end{array}$ & $\begin{array}{l}\tilde{O} \\
0 \\
0\end{array}$ & \begin{tabular}{|l|} 
\\
$\stackrel{y}{0}$ \\
0 \\
0
\end{tabular} & $\begin{array}{l}0 \\
\tilde{0} \\
0 \\
0 \\
0\end{array}$ & & & & & & & & & & & & & & & & & \\
\hline 14 & 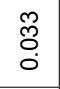 & $\begin{array}{l}\infty \\
\tilde{O} \\
0 \\
0\end{array}$ & 芯 & $\begin{array}{l}\mathscr{m} \\
\tilde{o} \\
0 \\
0\end{array}$ & $\begin{array}{c}\infty \\
\tilde{\delta} \\
0 \\
0\end{array}$ & $\begin{array}{l}\mathscr{N} \\
0 \\
0\end{array}$ & $\begin{array}{l}\infty \\
\tilde{O} \\
0 \\
0\end{array}$ & $\begin{array}{l}\mathscr{N} \\
0 \\
0\end{array}$ & $\begin{array}{l}\infty \\
\tilde{O} \\
0 \\
0\end{array}$ & $\begin{array}{l}\infty \\
\mathscr{O} \\
0 \\
0\end{array}$ & 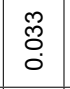 & $\begin{array}{l}\mathscr{N} \\
\stackrel{\delta}{0} \\
0\end{array}$ & $\begin{array}{l}\tilde{O} \\
0 \\
0\end{array}$ & & & & & & & & & & & & & & & & \\
\hline 15 & 0 & 0 & 0 & 0 & 0 & 0 & 0 & 0 & 0 & 0 & 0 & 0 & $\begin{array}{l}\infty \\
\stackrel{\infty}{0} \\
0 \\
0\end{array}$ & $\begin{array}{l}\tilde{m} \\
\stackrel{m}{0} \\
0\end{array}$ & & & & & & & & & & & & & & & \\
\hline 16 & $\begin{array}{l}m \\
\tilde{O} \\
0 \\
0\end{array}$ & $\begin{array}{l}\tilde{m}_{0} \\
0 \\
0 \\
0\end{array}$ & $\begin{array}{l}\mathscr{m} \\
\stackrel{0}{0} \\
0\end{array}$ & 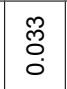 & $\begin{array}{l}\tilde{m}_{0} \\
\tilde{O} \\
0\end{array}$ & 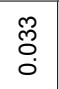 & $\begin{array}{l}\tilde{m}_{0} \\
0 \\
0\end{array}$ & $\begin{array}{l}\tilde{m} \\
\stackrel{0}{0} \\
0\end{array}$ & $\begin{array}{l}\tilde{m}_{0} \\
0 \\
0\end{array}$ & $\begin{array}{l}\tilde{m}_{0} \\
0 \\
0\end{array}$ & 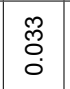 & 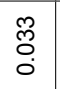 & - & - & 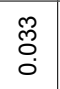 & & & & & & & & & & & & & & \\
\hline 17 & 0 & 0 & 0 & 0 & 0 & 0 & 0 & 0 & 0 & 0 & 0 & 0 & $\begin{array}{l}\mathscr{m} \\
\substack{0 \\
0}\end{array}$ & 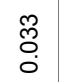 & 0 & $\begin{array}{l}\mathscr{m} \\
\stackrel{0}{0} \\
0\end{array}$ & & & & & & & & & & & & & \\
\hline 18 & 0 & 0 & 0 & 0 & 0 & 0 & 0 & 0 & 0 & 0 & 0 & 0 & $\begin{array}{l}\tilde{m} \\
\tilde{0} \\
0 \\
0\end{array}$ & $\begin{array}{l}\tilde{m} \\
\tilde{0} \\
0 \\
0\end{array}$ & 0 & $\begin{array}{l}\tilde{m} \\
\stackrel{n}{0} \\
0\end{array}$ & 0 & & & & & & & & & & & & \\
\hline 19 & $\begin{array}{l}\text { L̊ } \\
0 \\
0\end{array}$ & 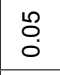 & 号 & \begin{tabular}{|l|}
$\stackrel{o}{0}$ \\
0 \\
0
\end{tabular} & $\begin{array}{l}0 \\
0 \\
0\end{array}$ & $\begin{array}{l}0 \\
0 \\
0\end{array}$ & 。̊. & $\begin{array}{l}0 \\
0 \\
0\end{array}$ & 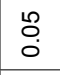 & 号 & $\begin{array}{l}0 \\
0 \\
0\end{array}$ & $\begin{array}{l}0 \\
0 \\
0\end{array}$ & - & - & $\begin{array}{l}0 \\
0 \\
0\end{array}$ & $\begin{array}{l}\text { to } \\
0\end{array}$ & $\begin{array}{l}\circ \\
0 \\
0\end{array}$ & $\begin{array}{l}\text { டி } \\
0 \\
\end{array}$ & & & & & & & & & & & \\
\hline 20 & $\begin{array}{l}\dddot{O} \\
\stackrel{0}{0} \\
0\end{array}$ & $\begin{array}{l}\infty \\
\tilde{O} \\
0 \\
0\end{array}$ & 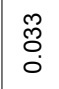 & $\begin{array}{l}\tilde{m} \\
\stackrel{0}{0} \\
0\end{array}$ & $\begin{array}{l}\text { } \\
\stackrel{\tilde{O}}{0} \\
0\end{array}$ & $\begin{array}{l}\dddot{m} \\
\stackrel{0}{0} \\
0\end{array}$ & 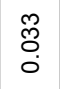 & $\begin{array}{l}\dddot{m} \\
\stackrel{0}{0} \\
0\end{array}$ & 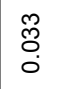 & $\begin{array}{l}\dddot{m} \\
\stackrel{0}{0} \\
0\end{array}$ & $\begin{array}{l}\tilde{y} \\
0 \\
0 \\
0\end{array}$ & $\begin{array}{l}\stackrel{\infty}{0} \\
\stackrel{0}{0}\end{array}$ & - & - & $\begin{array}{c}\mathscr{O} \\
0 \\
0\end{array}$ & o & $\begin{array}{l}\dddot{m} \\
\stackrel{0}{0} \\
0\end{array}$ & 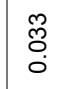 & do & & & & & & & & & & \\
\hline 21 & 0 & 0 & 0 & 0 & 0 & 0 & 0 & 0 & 0 & 0 & 0 & 0 & $\begin{array}{l}\tilde{m} \\
\tilde{O} \\
0 \\
0\end{array}$ & $\begin{array}{l}\tilde{m} \\
\tilde{o} \\
0 \\
0\end{array}$ & 0 & $\begin{array}{l}\tilde{m} \\
\tilde{o} \\
0 \\
0\end{array}$ & 0 & 0 & 号 & \begin{tabular}{|l|}
$\tilde{y}$ \\
0 \\
0
\end{tabular} & & & & & & & & & \\
\hline 22 & $\begin{array}{l}L_{0} \\
0 \\
0\end{array}$ & $\begin{array}{l}0 \\
0 \\
0\end{array}$ & 号 & $\begin{array}{ll}\stackrel{2}{0} \\
0 \\
0\end{array}$ & $\begin{array}{l}0 \\
0 \\
0\end{array}$ & 号 & 号 & 号 & $\stackrel{\leftrightarrow}{\circ}$ & 号 & $\begin{array}{l}\qquad \\
0 \\
0\end{array}$ & $\begin{array}{l}u_{0} \\
0 \\
0\end{array}$ & - & - & $\begin{array}{l}0 \\
0 \\
0\end{array}$ & $\begin{array}{l}\text { Oे } \\
\circ\end{array}$ & $\begin{array}{l}0 \\
0 \\
0\end{array}$ & 足 & 0 & $\begin{array}{l} \\
0 \\
0\end{array}$ & ஜ̊ & & & & & & & & \\
\hline 23 & 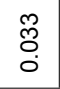 & $\begin{array}{l}\infty \\
\tilde{O} \\
0 \\
0\end{array}$ & $\begin{array}{l}\text { m } \\
\stackrel{0}{0} \\
0\end{array}$ & 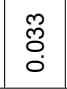 & 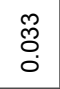 & $\begin{array}{l}\text { Oo. } \\
0 \\
0\end{array}$ & 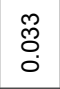 & $\begin{array}{l}\text { Oo. } \\
0 \\
0\end{array}$ & $\begin{array}{l}\text { Oొ } \\
\stackrel{\tilde{O}}{0} \\
0\end{array}$ & $\begin{array}{l}\dddot{0} \\
\stackrel{0}{0} \\
0\end{array}$ & $\begin{array}{l}\tilde{m} \\
0 \\
0 \\
0\end{array}$ & $\begin{array}{l}\tilde{m} \\
0 \\
0 \\
0\end{array}$ & - & - & $\begin{array}{l}0 \\
0 \\
0 \\
0\end{array}$ & o & $\begin{array}{l}\dddot{m} \\
0 \\
0\end{array}$ & $\begin{array}{l}\text { O్ } \\
0 \\
0 \\
0\end{array}$ & to & $\begin{array}{l}\tilde{O} \\
\stackrel{0}{0}\end{array}$ & do & do & & & & & & & \\
\hline 24 & 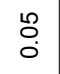 & 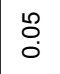 & 号 & 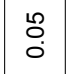 & 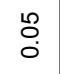 & $\stackrel{\text { ஜ̊ }}{0}$ & ஜ̊ & $\stackrel{\text { ஜ̊ }}{0}$ & $\stackrel{\mathscr{2}}{0}$ & 号 & $\begin{array}{l}\stackrel{2}{0} \\
0 \\
0\end{array}$ & $\begin{array}{l}\text { L̊ } \\
0 \\
0\end{array}$ & - & - & $\begin{array}{l}\text { ஜ̊ } \\
0\end{array}$ & $\stackrel{\Delta}{0}$ & $\begin{array}{l}n \\
0 \\
0 \\
0\end{array}$ & 号 & 0 & $\begin{array}{l}0 \\
0 \\
0\end{array}$ & $\stackrel{\text { L }}{0}$ & 0 & $\begin{array}{l}0 \\
0 \\
0\end{array}$ & & & & & & \\
\hline 25 & $\stackrel{l}{0}$ & 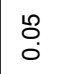 & 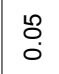 & \begin{tabular}{ll|} 
\\
0 \\
0 \\
0
\end{tabular} & $\mathscr{l}_{0}$ & 另 & $\mathscr{l}_{0}^{\circ}$ & 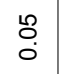 & $\stackrel{\leftrightarrow}{0}$ & 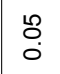 & $\begin{array}{l}\text { L̊ } \\
0 \\
0\end{array}$ & $\begin{array}{l}\mathscr{0} \\
0 \\
0\end{array}$ & - & - & $\begin{array}{l}0 \\
0 \\
0\end{array}$ & ¿̊. & 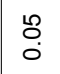 & 兽 & 0 & \begin{tabular}{l|} 
\\
0 \\
0
\end{tabular} & $\begin{array}{l}\text { 용 } \\
0\end{array}$ & 0 & $\begin{array}{ll} \\
0 \\
0\end{array}$ & 0 & & & & & \\
\hline 26 & 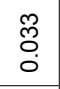 & $\begin{array}{l}\tilde{O} \\
0 \\
0 \\
0\end{array}$ & $\begin{array}{l}\dddot{m} \\
0 \\
0 \\
\end{array}$ & 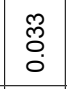 & 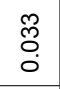 & ֶֻ, & $\begin{array}{l}\text { mo } \\
\text { Oo. } \\
0\end{array}$ & $\begin{array}{c}\tilde{o} \\
0 \\
0\end{array}$ & $\begin{array}{l}\tilde{O} \\
\stackrel{0}{0} \\
0\end{array}$ & $\begin{array}{l}\text { ల్ } \\
0 \\
0\end{array}$ & $\begin{array}{l}\tilde{0} \\
0 \\
0 \\
0\end{array}$ & $\begin{array}{l}\tilde{m} \\
\tilde{\delta} \\
0 \\
0\end{array}$ & - & - & $\begin{array}{l}\tilde{o} \\
0 \\
0 \\
0\end{array}$ & o & $\begin{array}{l}\tilde{O} \\
0 \\
0 \\
0\end{array}$ & \begin{tabular}{|l|}
0 \\
0 \\
0 \\
0
\end{tabular} & $\begin{array}{l}\text { to } \\
\text { Oे }\end{array}$ & $\begin{array}{l}0 \\
0 \\
0\end{array}$ & $\begin{array}{l}\tilde{y} \\
\stackrel{\tilde{o}}{0} \\
0\end{array}$ & to & $\begin{array}{l} \\
\delta \\
O \\
\end{array}$ & $\begin{array}{l}\Delta \\
0 \\
0\end{array}$ & 兽 & & & & \\
\hline 27 & $\begin{array}{l}\mathscr{L}^{\circ} \\
0 \\
0\end{array}$ & $\begin{array}{l}\qquad \\
0 \\
0\end{array}$ & $\begin{array}{l}{ }_{0} \\
0 \\
0\end{array}$ & $\begin{array}{l}\stackrel{\circ}{0} \\
0\end{array}$ & 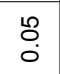 & $\begin{array}{l}\mathscr{0} \\
0 \\
0\end{array}$ & $\begin{array}{l}\mathscr{2} \\
0 \\
0\end{array}$ & 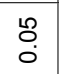 & 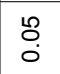 & 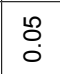 & $\begin{array}{l} \\
0 \\
0\end{array}$ & $\begin{array}{l}\text { L̊ } \\
0 \\
0\end{array}$ & - & - & $\begin{array}{l}0 \\
0 \\
0 \\
0\end{array}$ & $\begin{array}{l}\dot{0} \\
0 \\
0\end{array}$ & $\begin{array}{l}\stackrel{2}{0} \\
0 \\
0\end{array}$ & 号 & 0 & $\begin{array}{l} \\
0 \\
0\end{array}$ & $\begin{array}{l}\mathscr{l}^{2} \\
0 \\
0\end{array}$ & 0 & $\begin{array}{l}0 \\
0 \\
0\end{array}$ & 0 & 0 & $\begin{array}{l}0 \\
0 \\
0\end{array}$ & & & \\
\hline 28 & $\begin{array}{l}\mathscr{m} \\
\stackrel{0}{0} \\
0\end{array}$ & $\begin{array}{l}\infty \\
\tilde{O} \\
0 \\
0\end{array}$ & $\begin{array}{c}\tilde{m} \\
\stackrel{0}{0} \\
0\end{array}$ & $\begin{array}{l}\tilde{m} \\
\stackrel{\tilde{O}}{0} \\
0\end{array}$ & $\begin{array}{l}\tilde{m} \\
\tilde{o} \\
0 \\
0\end{array}$ & 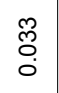 & $\begin{array}{l}\tilde{m} \\
\tilde{o} \\
0 \\
0\end{array}$ & 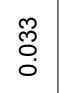 & $\begin{array}{l}\infty \\
\tilde{O} \\
0 \\
0\end{array}$ & $\begin{array}{l}\tilde{m} \\
\stackrel{0}{0} \\
0\end{array}$ & $\begin{array}{l}m \\
\tilde{o} \\
0 \\
0\end{array}$ & 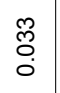 & 0 & 0 & 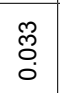 & - & $\begin{array}{l}\infty \\
\tilde{O} \\
0 \\
0\end{array}$ & $\begin{array}{l}\tilde{m} \\
0 \\
0 \\
0\end{array}$ & - & - & $\begin{array}{l}\tilde{m} \\
\tilde{O} \\
0 \\
0\end{array}$ & - & - & - & - & - & - & & \\
\hline 29 & - & - & - & - & - & - & - & - & - & - & - & - & $\begin{array}{l}8 \\
0 \\
0\end{array}$ & $\stackrel{8}{0}$ & - & $\stackrel{8}{0}$ & - & - & - & $\begin{array}{l}8 \\
0 \\
0\end{array}$ & - & - & $\stackrel{0}{0}$ & - & - & $\begin{array}{l}8 \\
0 \\
0\end{array}$ & - & $\stackrel{8}{0}$ & \\
\hline 30 & - & - & - & - & - & - & - & - & - & - & - & - & $\begin{array}{l}8 \\
0 \\
0\end{array}$ & $\stackrel{8}{0}$ & - & $\stackrel{8}{0}$ & - & - & - & $\begin{array}{l}0 \\
0 \\
0\end{array}$ & - & - & $\begin{array}{l} \\
\\
0\end{array}$ & - & - & $\begin{array}{l}8 \\
0 \\
0\end{array}$ & - & $\begin{array}{l}8 \\
0 \\
0\end{array}$ & 0 \\
\hline
\end{tabular}

Table 3: Genetic distance of 30 individuals of M. cuchia. 
cluster 2 which was comparatively nearer to cluster 1 . Clade 5 (S17, S28), clade 7 (S24, S26) and clade 8 (S29, S30) were very close to their respective pairs and their linkage distance was observed between 4-4.5 except clad $6($ S19, S27) which was around 4.6. All these clades were located under the cluster of 3 and formed cluster 4 when connected with cluster 2. S14 and S23 were found very similar to each other as well as S2, S18 and their linkage distances were seen 4.7 and 4.9 respectively. S23 was also found connected to the clade 9 (S9, S14) with a linkage distance of 5, 2, clade 9 and S23 were placed under the cluster of 5 which was also connected to the cluster 3 and 4 . Clade 10 was also linked to the cluster 5 . The most distally related samples were S8, S12 which produced a linkage distance of around 5.3 and they are connected through the most outer cluster, indicated cluster 6 .

\section{RFLP based genetic diversity}

Analysis of RFLP bands: PCR products of glutamine synthetase gene of freshwater mud eel, $M$. cuchia were digested with enzymes + CfrI and + Hpy178III and polymorphism was detected among the 30 individuals. These two enzymes have a single suitable restriction site to digest the glutamine synthetase gene of M. cuchia and a PCR amplified DNA was used without enzyme as a control to check proper enzyme activities, and this DNA did not cut and was found as a strong band on the gel indicating approximately $541 \mathrm{bp}$ of the glutamine synthetase gene. 25 individuals were digested by the enzyme $+\mathrm{CfrI}$ and approximately $280 \mathrm{bp}$ and $254 \mathrm{bp}$ long fragments of DNA were found, comparing with $1 \mathrm{~kb}$ plus DNA ladder where the marker was started with $250 \mathrm{bp}$. These 2 fragments indicating the wild type homogygotes and rest of the 5 individuals were found uncut with single band by the + CfrI that is indicating the polymorphic homozygote. The same PCR products of glutamine synthetase gene were again digested by the enzyme + Hpy178III where 19 individuals were digested with two bands of $343 \mathrm{bp}$ and $192 \mathrm{bp}$ lengths indicating wild type homogygotes. The uncut DNA fragments with 6 individuals showed 541 bp lengths indicating polymorphic homozygote. Remaining 5 individuals with 3 bands e.g., $541 \mathrm{bp}, 343 \mathrm{bp}$ and $192 \mathrm{bp}$ lengths were digested remark as heterozygocity.

\section{Observed genetic diversity}

Using the number of RFLP bands, proportions of shared fragments (F-value) were calculated from 30 individuals of freshwater mud eel where 7 different F-values such as $0.00,0.4,0.45,0.5,0.57,0.67$ and 1 were found. Using these F-values, genetic distances ( $\mathrm{p}$-value) were calculated and 7 different $p$-values such as $0.00,0.02,0.033,0.04,0.05$, 0.06 and 1 were observed (Table 3 ). On the basis of genetic distances, four different groups of individuals were identified. 16 individuals were found in one group with zero (0) p-value. Second group with seven individuals were observed with p-value of 0.033 . The third group with five individuals was recorded with p-value of 0.05 and finally, the fourth group with two individuals was recorded with $p$-value of 1 . The genetic distance between individuals in group one and group two was found at p-value 0.02 which means they are closely related. Second group were also found at short genetic distance $(\mathrm{p}=0.04)$ with third group. The genetic distance between the individuals of third and fourth group was found 0.06 , with large distance. Genetic distance between group one and three $(\mathrm{p}=0.04)$ as well as between group one and four $(\mathrm{p}=0.06)$ were found large distances and they are not closely related with each other.

\section{Discussion}

In this study, RAPD and RFLP based genotyping were performed to observe genetic status of freshwater mud eel, M. cuchia in Bangladesh. Using RAPD assay, in total 735 bands with 228 polymorphic loci was detected and polymorphisms were revealed by all the primers. Two previously conducted studies on the genetic diversity of freshwater mud eel, $M$. cuchia using three RAPD primers has reported higher genetic diversities alike the findings of this work $[18,24]$. Experimental data indicates that intra-specific polymorphism was observed $100 \%$ in this study; however, previous works has reported polymorphism with monomorphic loci as well $[18,24,35]$. Yin et al. [36] assessed the genetic differentiation and variation of the wild and raised swamp eels M. albus using RAPD technique and the results showed the percent polymorphic loci was $44.79 \%$ and $36.5 \%$, while, in a different work, the percent polymorphic loci of wild samples was found to be $60.6 \%$ to $71 \%$ range and cultured samples was found to be in the $54 \%$ to $56.3 \%$ range by ISSR analysis [23]. The findings of this result disagree with the findings of [36], Wei et al. [35], Alam et al. [6], Li et al. [23], Miah et al. [18] and Miah et al. [24], while lowest polymorphism was observed there. Though little bit higher polymorphism was found by Alam et al. [6] in a population of freshwater mud eel in Bangladesh and Ruzainah et al. [35] also found higher genetic diversity with RAPD fingerprinting of two loaches, Pangio piperata and Pangio filinaris. But this work has come with the higher genetic diversity among all the previously conducted works. In the rice field eel (Monopterus albus), 30 microsatellites were analyzed by AFLP of sequences where 13 loci exhibited polymorphism and these loci should provide a sufficient level of genetic variation [37], while lots of polymorphic loci were recorded in the present study of M. cuchia by RAPD assay. The genetic diversity of Asian swamp eel Monopterus albus were analyzed with 16 polymorphic novel microsatellites with 11 loci [35] which was also lowest from the present study in $M$. cuchia by RAPD analysis. In this study, inter individual pair-wise similarity of freshwater mud eel were recorded; we found 13 different groups where highest and lowest similarity was 14 and 1 respectively, which are a bit different from the reports of Miah et al. [18] and Miah et al. [24]. Highest genetic diversity recorded in this study refers almost similar inference with the reports of Miah et al [18], and Miah et al. [24]. Nei's genetic similarities were found identical to the report of Miah et al. [18] and Miah et al. [24]. Several values of linkage distances were found which ranges from 3.6 to 6.24, however, higher linkage distances were recorded in other populations by Miah et al. [18], Miah et al. [20]. A genetic relationship was observed through cluster analysis where 6 clusters were generated by 11 clades. Overall, higher genetic diversity has been recorded in this experimental using RAPD assay, which indicates the good genetic status of this fish in experimental nature. Miah et al. [18] found less genetic variation than the present study, but in another work, it was found higher [20].

In this study, partial sequence of glutamine synthetase gene of freshwater mud eel, M. cuchia was first time digested with two restriction enzymes and polymorphism was detected in an approximate size of 541 bp considering wild type homozygotes, polymorphic homozygotes and heterozygosity as well. The genetic diversity was observed with four different groups of individuals were identified and different genetic distances were recorded among the groups. The RFLP analysis of genetic polymorphism of glutamine synthetase gene was recorded higher than the findings of several previously reported studies [38]. Li et al. [15] analyzed hepcidin gene and they reported polymorphism in rice field $M$. albus. Tok et al. [37] analyzed glutamine accumulation and up-regulation of glutamine synthetase activity in the swamp eel, $M$. albus, however, no genetic diversity of glutamine synthetase gene of M. albus was studied. However, polymorphism was found among the population which is good for the experimental populations, though induced expression of downstream luc gene activities of Monopterus albus and Channa straitus were analyzed but no genetic diversity of this gene was analyzed [39]. 
Citation: Md. Miah F, Anwar S, Md. Ali H, Naser MN, Ahmed K (2018) Assessment of Genetic Diversity of Freshwater Mud Eel (Monopterus cuchia) Using RAPD and RFLP Markers. J Aquac Res Development 9: 543. doi: 10.4172/2155-9546.1000543

\section{Conclusion}

Higher genetic diversity has been recorded in this study by RAPD and RFLP observation which means the good genetic status in experimental habitat. However, more studies are essential to reveal the most specified genetic status of this fish considering different genomic analysis in detail at population level.

\section{Acknowledgements}

The work was almost fully funded by the research grant from Ministry of Science and Technology, Peoples republic of Bangladesh. The authors gratefully acknowledge the co-operation of the Department of Genetic Engineering and Biotechnology, Shahjalal University of Science and Technology, Sylhet 3114 Bangladesh and the Department of Zoology, University of Dhaka, Dhaka 1000, Bangladesh.

\section{References}

1. Hamilton F (1822) An account of the fishes found in the river Ganges and its branches. Printed for a Constable and company.

2. Ahmed GU, Akter N, Nipa SA, Hossain MM (2009) Investigation on health condition of a freshwater eel, Monopterus cuchia from Ailee beel, Mymensingh, Bangladesh. J Bangladesh Agril Univ 7: 421-426.

3. Atif AB, Zahri MK, Esa AR, Zilfalil BA, Rao USM, et al. (2015) Comparative analysis of the antibacterial, antifungal, antiproliferative and cyclic response element (CRE) induced expression of downstream luc gene activities of Monopterus albus and Channa straitus extracts. J Appl Pharm Sci 5: 42-47.

4. Do E (2015) Biodiversity National Assessment: Fifth National Report to the Convention on Biological Diversity: 147p.

5. He S, Liu X, Guo Z, Jin H, Zhang J (2004) On the genetic diversity of three species of Monopterus. J Hunan Agri Univ 30: 145-147.

6. Alam MS, Islam MS, Alam MS (2010) DNA fingerprinting of the freshwater Mud Eel, Monopterus cuchia (Hamilton) by randomly amplified polymorphic DNA (RAPD) marker. Int J Biochem Biotechnol 6: 271-278.

7. Eisenberg D, Almassy RJ, Janson CA, Chapman MS, Suh SW, et al. (1987) Some evolutionary relationships of the primary biological catalysts glutamine synthetase and RuBisCO. Cold Spring Harb Symp Quant Biol 52: 483-490.

8. Li W, Liao T, Yu XL, Cheng XM, Tong J (2007) Isolation and characterization of polymorphic microsatellites in a sex-reversal fish, rice field eel (Monopterus albus). Mol Ecol Resour 7: 705-707.

9. Hasan MM, Sarker BS, Nazrul KS, Rahman MM, Mamun AA (2012) Marketing channel and export potentiality of freshwater mud eel (Monopterus cuchia) of Noakhali region in Bangladesh. Int J Life Sc Bt Pharm Res 1: 226-233.

10. Hossian MS, Mazid MA (1995) A manual on development of floodplain fisheries. Fisheries Res Institute Mymensingh 2: 1

11. Hughes GM, Singh BR, Thakur RN, Munshi JSD (1974) Areas of the air-breathing surfaces of Amphipnous cuchia (Ham.). Proc Natl Acad Sci 40: 379-392.

12. Munjurul Hannan SM, Monirul HM, Rashiduzzaman A, Banu JN, Shameem $P$ et al. (2000) Red book of threatened fishes of Bangladesh. IUCN Bangladesh.

13. Lei L, Feng L, Jian TR, Yue GH (2012) Characterization and multiplex genotyping of novel microsatellites from Asian swamp eel, Monopterus albus. Conserv Genet Resour 4: 363-365.

14. Li WT, Liao XL, Yu XM, Cheng L, Tong J (2007) Isolation and characterization of polymorphic microsatellites in a sex-reversal fish, rice field eel (Monopterus albus). Mol Ecol Resour 7: 705-707.

15. Li W, Sun WX, Tang F, Li CP, Liu CD (2011) Molecular characterization and expression analysis of a hepcidin gene from rice field eel (Monopterus albus). Afr J Biotechnol 10: 7953-7961.

16. Liem KF (1967) Functional morphology of the integumentary, respiratory, and digestive systems of the synbranchoid fish Monopterus albus. Copeia 1976 375-388.
17. Lu DY, Song P, Chen YG, Peng MX, Gui JF (2005) Expression of gene vasa during sex reversal of Monopterus albus. Acta Zoological Sinica, 51: 469-475.

18. Miah FM, Haque F, Mia MR, Jannat E, Ali H, et al. (2013a) Molecular identification and sexual differentiation of freshwater mud eel, Monopterus cuchia. Univers J Agric Res 1: 54-58.

19. Li W, Sun WX, Meng L, Hong DW (2014) Molecular cloning, genomic structure, polymorphism and expression analysis of major histocompatibility complex class IIA gene of swamp eel Monopterus albus. Biologia 69: 236-246.

20. Miah MF, Ali H, Zannath E, Shuvra TM (2015) Breeding biology and induced breeding status of freshwater mud eel, Monopterus cuchia. Int J Inno Sci Res 9: 633-637.

21. Miah MF, Hafij Ali EJ, Naser MN, Ahmed MK (2015) Rearing and production performance of freshwater mud eel, Monopterus cuchia in different culture regimes. Advan Zool Bot 3: 42-49.

22. Miah MF, Naser MN, Ahmed MK (2015) The freshwater mud eel, Monopterus cuchia-A review. J Glob Bio0073ci 4: 1780-1794.

23. Li W, Sun WX, Fan J, Zhang CC (2013) Genetic diversity of wild and cultured swamp eel (Monopterus albus) populations from central China revealed by ISSR markers. Biologia 68: 727-732.

24. Miah MF, Zinnah KMA, Raihan MJ, Ali H, Naser MN (2016) Genetic diversity based population study of freshwater mud eel (Monopterus cuchia) in Bangladesh. World Acad Sci Eng Technol 10: 329-333.

25. Mishra N, Pandey PK, Datta Munshi JS, Singh BR (1977) Haematologica parameters of an air-breathing mud eel, Amphipnous cuchia (Ham) (Amphipnoidae; Pisces). J Fish Biol 10: 567-573.

26. Nasar SST (1997) Backyard eel culture.

27. Narejo NT, Rahmatullah SM, Rashid MM (2003) Length-weight relationship and relative condition factor $(\mathrm{Kn})$ of freshwater spiny eel, Mastacembelus armatus (Lacepede) from Mymensingh, Bangladesh. Indian J Fish 50: 81-87.

28. Narejo NT, Rahmatullah SM, Rashid MM (2003) Effect of different shelters on growth, survival and production of freshwater mud eel, Monopterus cucitici (Hamilton) reared in cemented cisterns of BAU Mymensingh. Bangladesh Pak J Biol Sci 6: 1753-1757.

29. Khongsngi JL (2010) Study on adaptive strategies against ammonia toxicity in the amphibious mud eel (Amphipnous cuchia): p: 270.

30. MoEF (2005) Biosafety Guidelines of Bangladesh.

31. Shafi M, Quddus MAA (1982) Bangladesher Matshya Sampad. Bangla Academy, Dhaka. p: 44.

32. Rahman AA (1989) Freshwater fishes of Bangladesh. Zool Soc of Bangladesh

33. Talwar PK (1991) Inland fishes of India and adjacent countries. CRC Press India. 2: 1

34. Ruzainah A, Azizah S, Patimah I, Amirrudin A (2003) RAPD fingerprinting of the eel-loaches Pangio filinaris and Pangio piperata: Preliminary evaluation. Aquaculture Research 34: 959-965.

35. Wei R, Quu G, Song R (2006) Genetic diversity of rice field eel (Monopterus albus) in China based on RAPD analysis. Asian Fish Sci 19: 61

36. Yin S, Li J, Zhou G, Liu Y (2005) Population genetic structure of rice field ee (Monopterus albus) with RAPD markers. Chin J Appli Environ Biol 11: 328-332.

37. Tok CY, Chew SF, Peh WY, Loong AM, Wong WP, et al. (2009) Glutamine accumulation and up-regulation of glutamine synthetase activity in the swamp eel, Monopterus albus (Zuiew), exposed to brackish water. J. Exp. Biol 212: 1248-1258.

38. Yang FQ, Zhou QB, Zhang YP, Li XH (2005) Variation of esterase isoenzyme among three kinds of bodily color Monopterus albus in Poyang Lake Region. J Econ Anim 9: 110.

39. Zaher M, Mazid MA (1995) Aquafeeds and feeding strategies in Bangladesh FAO Fisheries Technical Paper. pp: 161-180. 